\title{
Octave spanning supercontinuum generation in InGaP waveguides on a silicon substrate at $1550 \mathrm{~nm}$
}

\author{
Utsav D. Dave, ${ }^{1,2, *}$ Bart Kuyken, ${ }^{1,2}$ Sylvain Combrie, ${ }^{3}$ Alfredo De Rossi, ${ }^{3}$ Fabrice Raineri,,${ }^{4,5}$ and Gunther \\ Roelkens ${ }^{1,2}$ \\ ${ }^{1}$ Photonics Research Group, Department of Information Technology, Ghent University-IMEC, Ghent B-9000 Belgium \\ ${ }^{2}$ Center for Nano- and Biophotonics (NB-photonics), Ghent University, Belgium \\ ${ }^{3}$ Thales Research and Technology, Route Départementale 128, 91767 Palaiseau, France \\ ${ }^{4}$ Laboratoire de Photonique et de Nanostructures, CNRS-UPR20, Route de Nozay, 91460 Marcoussis, France \\ ${ }^{5}$ Université Paris Denis Diderot, 75205 Paris, France \\ *utsav.dave@intec.ugent.be
}

\begin{abstract}
We present an octave spanning supercontinuum (at the $-35 \mathrm{~dB}$ level) ranging from 1100 $\mathrm{nm}$ to $2500 \mathrm{~nm}$ in InGaP photonic wire waveguides that are bonded to a silicon substrate and pumped by an ultrashort pulsed laser centered at $1550 \mathrm{~nm}$.
\end{abstract}

OCIS codes: (130.4310) Integrated optics, Nonlinear; (190.4400) Nonlinear optics, materials; (320.6629) Ultrafast optics, supercontinuum generation; (90.4390) Nonlinear optics, integrated optics.

\section{Introduction}

Supercontinuum sources provide broadband, spatially and/or temporally coherent radiation. These properties are useful for many applications such as spectroscopy, optical coherence tomography, wavelength division multiplexing etc. Indeed, for these applications wide bandwidth, intense and coherent spectra are desired. An octave spanning supercontinuum is desired when $f-2 f$ interferometric stabilization of a frequency comb is needed [1]. Photonic crystal fibers have been widely used for supercontinuum generation purposes because of the possibility to achieve a tailored dispersion profile which can be critical to achieving the widest possible supercontinuum [2]. An integrated optical approach to supercontinuum generation however, is interesting because of the potential to make these sources compact, low-cost, efficient and robust. Consequently, approaches for achieving supercontinuum generation in various on-chip platforms have been investigated, including silicon [3-5], silicon nitride [6] and chalcogenide [7] waveguide platforms. In general, a large nonlinear refractive index, combined with a large index contrast for achieving high intensities, low linear and nonlinear losses, and the possibility to engineer the right dispersion profile and a large transparency window within the wavelength range of interest are desirable. Silicon suffers from large nonlinear losses at the telecom wavelengths due to two-photon absorption and the associated free carrier absorption and is not transparent below $1100 \mathrm{~nm}$. Silicon nitride has a relatively low index contrast and nonlinearity. In contrast, certain III-V materials like AlGaAs and InGaP have large nonlinearities (comparable to or larger than silicon), a large refractive index comparable to silicon as well as no nonlinear losses from two-photon absorption at telecom wavelengths due to their larger bandgaps. This enables the use of widely available lasers in the telecom wavelengths as pump sources. Thus, III-V materials are very promising and they have been investigated by several groups for various nonlinear applications $[8,9]$.

We have previously reported a new platform for integrated nonlinear optical applications based on high index contrast InGaP waveguides on a silicon substrate which could enable a variety of integrated nonlinear devices [9]. Here we further demonstrate the efficacy of this approach for nonlinear applications by achieving an octave spanning supercontinuum ranging from $1100 \mathrm{~nm}$ to $2500 \mathrm{~nm}$ in $2 \mathrm{~mm}$ long waveguides. In section 2 we discuss in brief the process flow for fabricating the InGaP waveguides on a silicon substrate; in section 3 we present the results of the supercontinuum generation and in section 4 we discuss the future outlook and conclusions.

\section{Fabrication process}

Figure 1 summarizes the process for fabricating the InGaP waveguides and partially etched grating couplers on a silicon substrate. The InGaP layer (grown lattice matched to a GaAs substrate with a $1400 \mathrm{~nm}$ thick GaAs sacrificial layer and a $200 \mathrm{~nm}$ thick InGaP etch stop layer in between) is first bonded using Benzocyclobutene (BCB) to a silicon substrate that has a $3 \mu \mathrm{m}$ thermal oxide layer on top. Before bonding, $200 \mathrm{~nm}$ of oxide is deposited using plasma enhanced chemical vapour deposition (PECVD) on the InGaP layer. The thickness of the BCB bonding layer can be controlled by its dilution and spinning rate - in this work we use a thick BCB layer of approximately $1 \mu \mathrm{m}$. The BCB is cured at a temperature of $300{ }^{\circ} \mathrm{C}$. The GaAs substrate and sacrificial layer are removed via wet etching in an oxidizing nitric acid solution of $\mathrm{HNO}_{3}: \mathrm{H}_{2} \mathrm{O}_{2}: \mathrm{H}_{2} \mathrm{O}$ in volume ratio of $1: 4: 1$. The InGaP etch stop layer is removed by a 1:1 volume mixture of $\mathrm{HCl}$ and $\mathrm{H}_{3} \mathrm{PO}_{4}$. The waveguides are defined by e-beam lithography and etched 
via inductively coupled plasma etching using a $\mathrm{HBr}: \mathrm{O}_{2}: \mathrm{He}$ gas mixture. Grating couplers are etched in a second etch step because they use a partial etch. The grating couplers are designed for TE polarized light at $1550 \mathrm{~nm}$ and have a $3 \mathrm{~dB}$ bandwidth of about $45 \mathrm{~nm}$. The waveguides have a linear loss of $12 \mathrm{~dB} / \mathrm{cm}$, which can be improved with further optimization of the etching process in the future. More details about the fabrication can be found in our previous work [9].

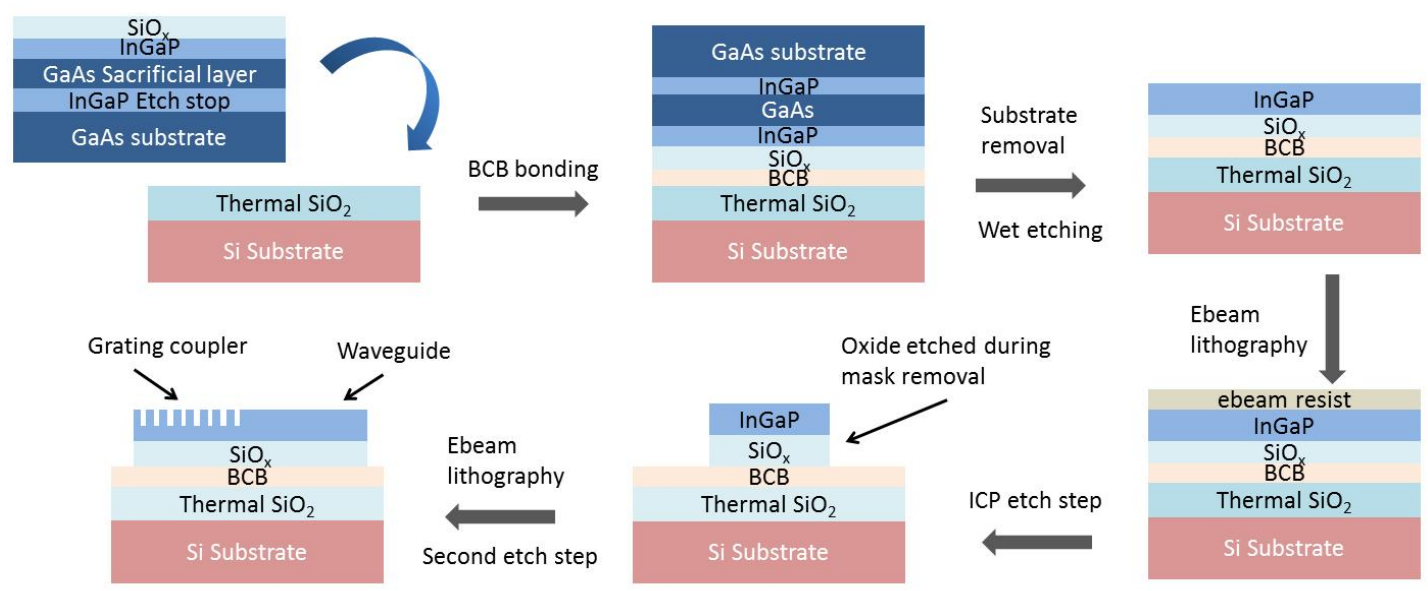

Fig. 1. The process flow for the fabrication of the InGaP waveguides and shallow etched grating couplers after bonding to a silicon substrate.

\section{Supercontinuum generation}

For the supercontinuum generation we pumped a $760 \mathrm{~nm}$ wide and $250 \mathrm{~nm}$ thick InGaP dispersion engineered waveguide with a fiber-based modelocked laser emitting a pulse train with a hyperbolic secant pulse shape and a pulse width of $76 \mathrm{fs}$ at the highest power level. The mechanism of the supercontinuum generation in the femtosecond regime is soliton fission due to the perturbation from higher order dispersion terms [1, 10]. The simulated second order dispersion profile of the waveguide is presented in Fig. 2 below, showing that the waveguide exhibits anomalous dispersion at the pump wavelength of $1550 \mathrm{~nm}$. The inset shows the waveguide cross-section where we see that the surrounding oxide around the waveguide has been etched by about $200 \mathrm{~nm}$, which is due to the fact that during the removal of the e-beam lithography mask, the unprotected oxide surrounding the waveguide is also etched.

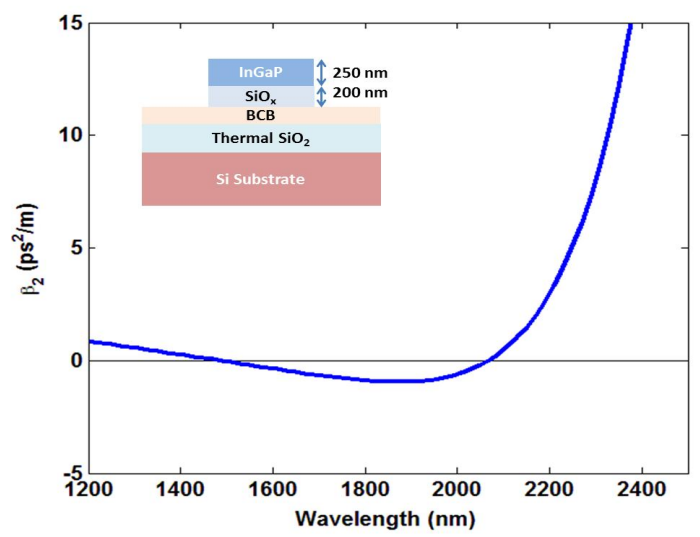

Fig.2. The simulated group velocity dispersion profile of the InGaP waveguide used in the supercontinuum generation experiment. Inset: Schematic of the waveguide cross-section.

The buildup of the supercontinuum with increasing coupled-in peak power is shown in fig. 3. As we reported in [9], these waveguides only suffer from nonlinear losses due to three-photon absorption rather than the much stronger two-photon absorption as would be the case in silicon at these wavelengths. Hence, we are able to pump these waveguides much harder without suffering from the adverse effects of nonlinear losses. At the $-35 \mathrm{~dB}$ level, the 
supercontinuum at the highest pump power $(770 \mathrm{~W})$ spans from $1200 \mathrm{~nm}$ to $2500 \mathrm{~nm}$ which is over an octave of bandwidth. With a different optical spectrum analyzer, it was observed that the spectrum extended down to 1100 $\mathrm{nm}$.

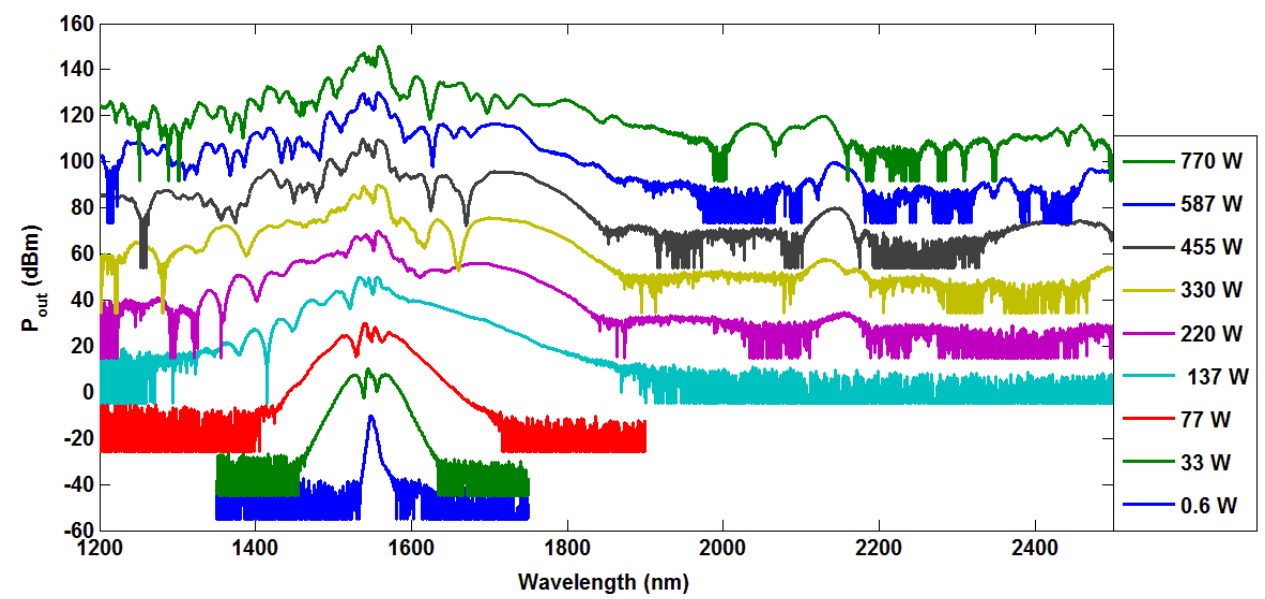

Fig. 3. The buildup of the supercontinuum with increasing pump powers - the spectrum at the highest pump power spans more than an octave of bandwidth at the $-35 \mathrm{~dB}$ level. Successive plots have been shifted by $20 \mathrm{~dB}$ for clarity.

\section{Future outlook and conclusions}

Apart from the bandwidth and power, another desired property of the supercontinuum is that it is coherent $[1,5]$. While several techniques for experimentally measuring the coherence of the supercontinuum exist, one of the easiest to implement is to interfere successive pulses of the supercontinuum [10]. The fringe depth of such an interference spectrum can then be related to the degree of coherence between the pulses and gives information about the coherence over the entire spectrum where fringes are observed. It would be instructive to investigate coherence properties of the supercontinuum presented here using this technique. Preliminary results indicate that the supercontinuum is coherent at least in the spectral region close to the pump - a more detailed investigation is underway. Further improvements through reduction of linear losses and dispersion engineering are also possible. These avenues will be explored in future works. This approach of highly nonlinear III-V waveguides on a silicon substrate can also be applied to second-order nonlinear effects since these III-V materials also possess large secondorder nonlinear coefficients. Finally, in order to exploit various functionalities developed in the SOI or the silicon nitride platform, these waveguides can be evanescently coupled to the SOI or nitride waveguides below, an approach already in wide use in heterogeneously integrated III-V lasers on the SOI platform.

In conclusion, we have demonstrated octave spanning supercontinuum generation in highly nonlinear InGaP waveguides bonded to a silicon substrate in the telecommunications wavelength range.

\section{References}

[1] R. Holzwarth, T. Udem, T. W. Hänsch, J. C. Knight, W. J. Wadsworth, and P. St. J. Russell, “Optical Frequency Synthesizer for Precision Spectroscopy," Phys. Rev. Lett. 85(11), 2264-2267 (2000).

[2] J. M. Dudley, G. Genty, and S. Coen, “Supercontinuum generation in photonic crystal fiber," Rev. Mod. Phys. 78, 1135-1184 (2006).

[3] I. W. Hsieh, X. Chen, X. Liu, J. I. Dadap, N. C. Panoiu, C. Y. Chou, F. Xia, W. M. Green, Y. A. Vlasov, and R. M. Osgood Jr., "Supercontinuum generation in silicon photonic wires," Opt. Express 15(23), 15242-15249 (2007).

[4] U. D. Dave, S. Uvin, B. Kuyken, S. Selvaraja, F. Leo, and G. Roelkens, "Telecom to mid-infrared spanning supercontinuum generation in hydrogenated amorphous silicon waveguides using a Thulium doped fiber laser pump source," Opt. Express, 21(26), 32032-32039 (2013).

[5] B. Kuyken, T. Ideguchi, S. Holzner, M. Yan, T. W. Hänsch, J. Van Campenhout, P. Verheyen, S. Coen, F. Leo, R. Baets, G. Roelkens, and N. Picqué, “An octave-spanning mid-infrared frequency comb generated in a silicon nanophotonic wire waveguide,” Nat. Commun. 6, 6310 (2015). [6] R. Halir, Y. Okawachi, J. S. Levy, M. A. Foster, M. Lipson, and A. L. Gaeta, "Ultrabroadband supercontinuum generation in a CMOScompatible platform,” Opt. Lett. 37(10), 1685-16587 (2012).

[7] M. R.E. Lamont, B. Luther-Davies, D-Y. Choi, S. Madden, and B. J. Eggleton, "Supercontinuum generation in dispersion engineered highly nonlinear $(\gamma=10 / \mathrm{W} / \mathrm{m})$ As2S3 chalcogenide planar waveguide," Opt. Express 16(19), 14938-14944 (2008).

[8] C. Lacava, V. Pusino, P. Minzioni, M. Sorel, and I. Cristiani, "Nonlinear properties of AlGaAs waveguides in continuous wave operation regime," Opt. Express 22(5), 5291-5298 (2014).

[9] U. D. Dave, B. Kuyken, F. Leo, S-P. Gorza, S. Combrie, A. De Rossi, F. Raineri, and G. Roelkens, "Nonlinear properties of dispersion engineered InGaP photonic wire waveguides in the telecommunication wavelength range," Opt. Express 23(4), 4650-4657 (2015).

$[10]$ F. Leo, S-P. Gorza, S. Coen, B. Kuyken, and G. Roelkens, "Coherent supercontinuum generation in a silicon photonic wire in the

telecommunication wavelength range," Opt. Lett. 40(1), 123-126 (2015). 